\title{
Workshop Report 'Responding to Challenges in Metastatic Breast Cancer - an Academy for Rising Stars in Oncology', 25th-27th January 2013, Lisbon
}

\author{
Maria Roethlisberger ${ }^{a, b}$ Lisa Richters ${ }^{a, b}$ Fatima Cardoso ${ }^{e} \quad$ Rachel Wuerstlein $^{c, d}$ \\ aDepartment of Obstetrics and Gynecology, Breast Center, University of Cologne; ${ }^{\mathrm{B}} \mathrm{ClO}$ Cologne-Bonn, Cologne; ${ }^{\mathrm{B} B r e a s t}$ Center, Department \\ of Obstetrics and Gynecology; 'Comprehensive Cancer Center, University of Munich, Germany; ${ }^{\mathrm{e} C h a m p a l i m a u d ~ C a n c e r ~ C e n t r e, ~ L i s b o n, ~}$ \\ Portugal
}

PrIME Oncology is a global independent medical education organisation focussing on specialist training for oncologists. Meetings are held in Europe and the US updating the physicians on the latest guidelines and treatments for malignancies. PrIME Oncology provides physicians with evidence based, state of the art educational activities to improve patient outcomes. The meeting 'Responding to Challenges in metastatic Breast Cancer' was held on January 25-27 in Lisbon. It was designed as a workshop consisting of 3 plenary sessions with updates on the most recent guidelines and 3 breakout sessions where discussions were held and presenting skills could be practised. The faculty consisted of the chair of the meeting, Fatima Cardoso, MD (Champalimaud Cancer Centre, Lisbon), Robert Coleman, MD, FRCP, FRCPE (University of Sheffield, US), Angelo Di Leo, MD, PhD (IstitutoToscanoTumori, Prato, Italy), Alessandra Gennari, MD, PhD (Galliera Hospital; Genua, Italy), Nadia Harbeck, $\mathrm{MD}, \mathrm{PhD}$ (University of Munich (LMU), Germany) and Frédérique Penault-Llorca, MD, PhD (Centre Jean Perrin, Université d'Allvergne Clermont, France). They were joined by Jilly Carter, head of Carter Communications and former BBC journalist as a non-medical expert. She shared her knowledge of presentation skills and interacting with the press. The 23 participants came from the UK, Belgium, France, Germany, Italy, Portugal and Greece. Except for the German participants, who were gynaecologists, all were medical oncologist with a 5-10-year experience in treatment of solid tumours. At the beginning, Fatima Cardoso gave an excellent overview about the last years' developments. Even though the diagnostics and treatment of patients with metastasized breast cancer, especially when treated according to recent guidelines, has substantially improved, overall survival still does not exceed 2-3 years, showing this disease cannot be called a chronic disease yet.

Therefore, the main focus of the meeting was biomarkers and translational research in personalized medicine in metastatic breast cancer. Especially endocrine responsive disease and new treatment concepts for this cancer type were discussed. Strengths, weaknesses, opportunities and threats of aromatase inhibitors, tamoxifen and fulvestrant as current therapeutic options were talked about. The SWOG S0226 trial was presented, showing a longer progression-free survival with the combination of anastrozole and fulvestrant which, however, seemed to be limited to patients without prior endorine therapy. The higher dose fulvestrant of $500 \mathrm{mg}$ was associated in the FIRST trial with a longer time to progression in metastatic endocrine responsive breast cancer. New targeted therapies affecting the mTOR pathway and recent results of the phase III study BOLERO 2 were presented, demonstrating a longer progression free survival of 4 months and even a trend to a better overall survival with everolimus. Moreover, the effect of everolimus on bone metastasis was highlighted by the GBG RADAR study data. Looking for a way to overcome endocrine resistance, further studies on mTOR/PI3K pathway inhibitors are planned, such as the BELLE trial programme. A new phase II trial with the CDK 4-6 Inhibitor PD 0332991 plus letrozole showing a better progression free survival than letrozole alone was also presented. The data for the FGF-R pathway as a therapeutic target are promising but still under investigation. Overall, combining endocrine and targeted therapeutics is a next step on our way to tailored medicine, but there is a need for biomarkers predicting therapy sensitivity.

Data of new chemotherapeutic options in metastatic breast cancer were also presented focusing on the palliative character of this treatment option. Using nanotechnology, albuminbound taxane (nab-paclitaxel) seems to be a good alternative to conventional taxanes. The EMBRACE study showed 2 months longer overall survival for eribulin compared to treatment of physician's choice. The new agent NKTR-102 which showed promising phase II data is now tested in the currently open BEACON trial. The new option of staging patients using new techniques such as molecular imaging (PET CT) was also discussed.

\section{KARGER}

Fax +497614520714

Information@Karger.com

www.karger.com
(C) 2013 S. Karger GmbH, Freiburg

Accessible online at:

www.karger.com/brc
Dr. Maria Röthlisberger

Klinik und Poliklinik für Frauenheilkunde und Geburtshilfe

Uniklinik Köln

Kerpener Str. 34

50931 Köln, Germany

maria.roethlisberger@uk-koeln.de 
The important role of the pathologist in the diagnosis of breast cancer was highlighted. Several trials showing changes in detection of biomarkers in tumour tissue depending on transport or fixation time emphasise the need of correct tissue handling for a proper diagnosis and addressed the question of quality improvement and standardization. Intratumoural heterogeneity and the change of biomarkers during progression of metastatic breast cancer were reviewed, highlighting the need for re-biopsy: 'The future is now!'

The second part of the meeting touched on the themes of presenting medical data and giving interviews. Jilly Carter shared valuable information from her BBC journalist experience. Main points were the right structure for a presentation with a strong beginning, a clear middle part and a memorable ending. The need for repetition of important points and for enough practice was emphasised. A clear and easy structure of power point slides was also demonstrated, avoiding 'death by power point'. In giving interviews, the need to illustrate and give examples was pointed out as well as the need to keep to one's key messages. A special method for giving short and to the point presentations, called 'The Elevator Presentation', was practised. Participants had to convince the audience of their point of view in 30 seconds.

The meeting also featured training in Oxford debate presented by participants, discussing the role of biomarkers in metastatic breast cancer. The supporters discussed the importance of re-biopsy when metastases are diagnosed. The opponents mentioned the difficulty in finding reliable markers and that treatment should not be delayed while searching for markers. Individual performances and suggestions for improvements were discussed afterwards. The importance of giving a passionate talk and entertaining the audience was especially pointed out.

Three breakout sessions were held, in which small groups of 6-8 participants separately discussed topics with 2 board members each. The first group talked about the organisation of a breast centre (chairs: Coleman and DiLeo). The importance of selecting one's collaborators was highlighted, as well as the need to work in a multidisciplinary team to optimise the treatment for each individual patient. Furthermore, the importance of collaborating with other hospitals, pharmaceutical companies and researchers was pointed out. Differences between the structures of breast centres in the countries of the different participants were discussed. Some of us had the opportunity, to visit Dr. Cardoso's new Champalimaud Cancer Center as an example for combining individual cancer care and personalized medicine with high standards of care and translational research. In the second breakout session (chairs: Harbeck and Penault-Llorca) the change of different biomarkers in the course of a breast cancer patient was discussed based on a case. The importance of re-evaluation of biomarkers in metastatic breast cancer was highlighted, especially in patients where the tumour was detected before 2005 and HER2 had not been routinely analysed. Different therapeutic options were discussed; also differences between therapeutic regimens in different countries. For instance, in the UK, trastuzumab is only reimbursed by the insurance companies for one line of treatment in metastatic breast cancer. The last breakout session was about presentation skills and writing abstracts for scientific meetings (chairs: Cardoso and Gennari). The chairpersons shared personal experience with presentations on big meetings. Based on abstracts and presentations of the participants the ideal structure of an abstract and a presentation was developed. Participants also had to give a talk without slides spontaneously and their performance was talked through.

This first Rising Stars in Oncology meeting focussing on metastatic breast cancer was a full success. During the whole meeting, participants had the chance to talk to the board members and received valuable tips for presenting scientific research at an international meeting and regarding further steps for their education and career. Further meetings are planned to support this international network of young oncologists in Europe. Therefore, on behalf of all participants, the authors want to thank all the organisers and chairpersons.

For further information see www.primeoncology.org.

Fig. 1. The chairs and participants.

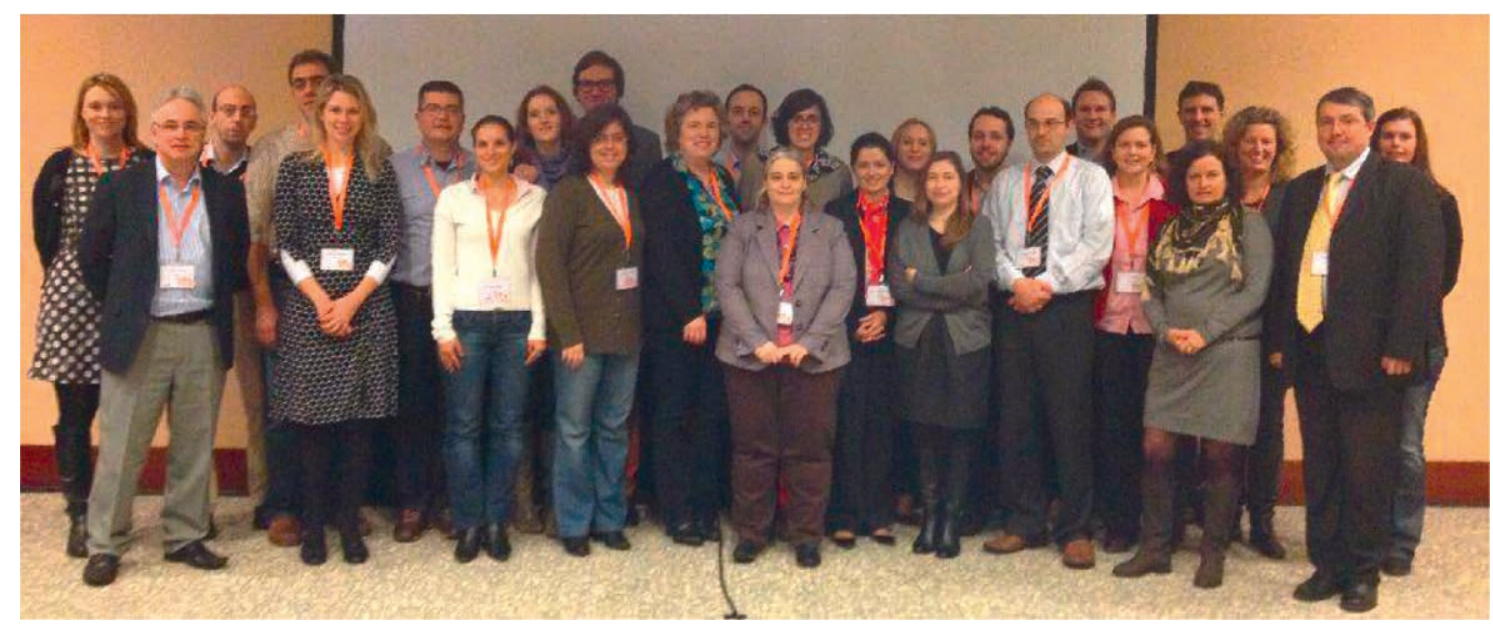

